production workers can be exposed to inhalational hazards such as diacetyl and 2,3-pentanedione, which are associated with the development of occupational respiratory disease, including obliterative bronchiolitis, a rare and irreversible lung disease.

Objective Identify determinants contributing to task-based exposures to alpha-diketones, specifically diacetyl and 2,3-pentanedione, at 17 U.S. coffee roasting and packaging facilities.

Methods We collected 606 personal task samples including roasting $(n=189)$, grinding $(n=74)$, packaging $(n=203)$, quality control $(n=44)$, flavoring $(n=15)$, and miscellaneous production/café $(n=81)$. Samples were collected and analyzed according to the modified OSHA Method 1013/1016. Information on sample-level and process-level factors relating to production scale, sources of alpha-diketones, and engineering controls was collected during surveys. Bayesian mixed-effect regression models accounting for censored data were fit for overall data and specific tasks including roasting, grinding, packaging, quality control, and flavoring. Significant determinants were used in multiple regression models using variable selection or model averaging Bayesian methods.

Results Task-based sample durations ranged from 2-86 minutes. Total number of alpha-diketone sources, sum of all open storage sources, average roasted coffee production per day, average percent of production as ground coffee, flavoring during survey, and number of grinders all resulted in increased exposures for at least one task category. General exhaust ventilation (GEV) with natural ventilation, supply air turned on, and GEV turned on resulted in lowered exposures for at least one task category.

Conclusions GEV and supply air turned on and natural ventilation were identified in most models as determinants which reduced exposures. Open storage of roasted coffee and flavoring during survey were significant in most models contributing to higher task exposures and can be targeted for exposure mitigation to reduce short-term and full-shift exposures and minimize risks for respiratory disease.

\section{0-137 ASSESSMENT OF MULTIPLE EXPOSURES TO CHEMICAL AGENTS IN FRENCH WORKPLACES: FINDINGS FROM TWO EXPOSURE DATABASES}

${ }^{1}$ Jean-Francois Sauve, Andrea Emili, Gautier Mater. ${ }^{1}$ Institut national de recherche et de sécurité (INRS), France

\subsection{6/OEM-2021-EPI.45}

Background National surveys of the French working population estimate that approximately $15 \%$ of all workers are exposed to at least three different chemical agents at work. However, the most prevalent coexposure situations and their associated health risks remain relatively understudied.

Objective To characterize occupational coexposure situations in France using available data from two occupational exposure databases.

Methods We extracted personal measurement data from the Colchic and Scola databases for the period 2010-2019. We selected 118 chemical agents that had $\geq 100$ measurements with detected concentrations, including 31 carcinogens (IARC groups 1, 2A, and 2B). We grouped measurements by work situation (WS, combination of sector, occupation, task, and year). We characterized the mixtures across WS using frequent itemset mining methods.

Results We retained 275,213 samples from 32,670 WS, encompassing 4,692 unique mixtures. Workers in thirty-two percent of all WS were exposed to $\geq 2$ agents (median 3 agents/WS, interquartile interval 2-5) and $13 \%$ of all WS contained $\geq 2$ carcinogens (median 2 carcinogens/WS, maximum 14). The most frequent coexposures across all agents were ethylbenzene-xylene (1,550 WS), quartz-cristobalite (1,417 WS), and toluene-xylene (1,305 WS). Prevalent combinations of carcinogens also included hexavalent chromiumlead (368 WS) and benzene-ethylbenzene (314 WS). Agents with the lowest proportions of coexposure were wood dust (6\% of WS exposed to at least one other agent) and asbestos $(8 \%)$. Tasks with the highest proportions of coexposure to carcinogens include electric arc welding (37\% of WS with coexposure), polymerization and distillation (34\%), construction drilling and excavating (34\%), and water collection and treatment $(32 \%)$.

Conclusion Coexposure to multiple chemical agents, including carcinogens, was highly prevalent in the databases, and should be taken into account when assessing exposure risks in the workplace. However, these databases do not necessarily represent a random sample of the working population, thereby limiting the generalizability of our findings.

\section{0-156 PESTICIDE EXPOSURE IN FRUIT GROWERS: COMPARISON OF LEVELS AND DETERMINANTS FROM THE CANEPA FIELD STUDY AND FROM THE 'AGRICULTURAL OPERATOR EXPOSURE MODEL' (AOEM) PREDICTIONS}

${ }^{1}$ Morgane Bresson, Mathilde Bureau, Jeremie Le Goff, Yannick Lecluse, Elsa Robelot, Isabelle Baldi, Pierre Lebailly. 'INSERM / Université Caen, France

\subsection{6/OEM-2021-EPI.46}

Introduction Pesticide exposures increase the risk of chronic diseases in farmers. Knowledge of exposure levels are needed for epidemiological studies and for regulation. In pesticide registration process, operator's exposure is predicted by the AOEM, set up in 2014 by the European Food Safety Authority, based on thirty studies conducted by the pesticide industry. To date, we are not aware of any field study comparing predicted data with those measured under real working conditions.

Objective We aimed to compare operators' exposures during treatment days in apple-growing under non-controlled conditions of work and values predicted by AOEM.

Methods Thirty apple growers from the French CANEPA study, were observed applying two fungicides (captan/dithianon) in 2016-2017. Dermal exposure was measured by body patches and cotton gloves. Detailed parameters about the farm, operator, application day, spraying equipment and personal protective equipment (PPE) used were recorded. For each observation, the corresponding exposure was calculated by the AOEM, using these parameters. The relationship between measured and calculated exposures was studied by linear regression.

Results Significant linear correlation was observed between calculated and measured daily exposures. Overall, the model 
overestimated daily exposure and exposure during application. However exposure was underestimated at mixing/loading in many observations, especially when the operator wore long working clothes or gloves.

Conclusion The AOEM model did not appear conservative in the sense that it did not overestimate exposures in all circumstances. More specifically: 1) the overestimation at spraying appeared a consequence of the overestimation of daily treated area, 2) the protection provided by PPE appeared overestimated, 3) mixing/loading exposure, a phase in which operators are exposed to concentrated products, appeared underestimated. These discrepancies could be due to optimal working conditions (larger farms, newer equipment) under which industries' studies are conducted that are not representative of operators' actual working conditions in fruit growing.

\section{0-185 ASSESSING OCCUPATIONAL AND ENVIRONMENTAL DEPLOYMENT-RELATED MILITARY EXPOSURE AMONG U.S. VETERANS}

1Paul D Blanc, Anna Korpak, Andrew Timmons, Karen Nakayama, Susan Proctor, Nicholas Smith, Eric Garshik. 'University of California San Francisco, United States

\subsection{6/OEM-2021-EPI.47}

Introduction Twenty-first century occupational and environmental (OE) exposures arising from military service encompass combustion byproducts, particulate matter, and traditional job exposures.

Rationale Multiple potentially collinear exposures can require data reduction to facilitate epidemiological analyses. Our data provided an opportunity to characterize relationships among a range of interrelated exposures.

Methods We analyzed interim data from the Veterans Affairs 'CSP \#595: Service and Health Among Deployed Veterans' study. As of May 2020, survey responses were available from 1962 randomly selected Veterans with one or more deployments to Afghanistan, Iraq, or elsewhere in southwest Asia between 2001 and 2017. The interviewer-administered questionnaire yielded 3-level responses to 32 OE deployment-associated exposures included in this analysis. We identified a priori six anticipated exposure factors: burn pit (4 items); other open combustion sources (5 items); combustion engine byproducts (5 items); mechanically generated dust/dust storms (4 items); occupational vapors, gas, dust, and fumes (VGDF; 11 items); and other toxicants (3 items). We used confirmatory factor analysis (CFA) to assess construct validity of these groupings.

Results In preliminary CFA, two of six groupings that were highly collinear (combustion engine byproducts/mechanically generated dust) required combination for model viability. The resulting 5 factor model performed adequately (incremental or Comparative Fit Index $[\mathrm{CFI}]=0.945)$. After excluding 4 of 32 items with lower factor loadings ( 0.50 for 28 3-level items on five factors.

Conclusion These findings provide a basis for item reduction in an expansive survey battery of exposure items addressing occupational and environmental military exposures. We identified 28 items (three response levels) comprising five distinct factors. These results suggest that among previously deployed Veterans, multiple OE exposures can be simplified to exposure-related factors as part of assessing health effects.

\section{0-262 IMPACT OF OCCUPATIONAL PESTICIDE EXPOSURE ASSESSMENT METHODS ON RISK ESTIMATES FOR PROSTATE CANCER, NON-HODGKIN'S LYMPHOMA AND PARKINSON'S DISEASE - RESULTS OF THREE META- ANALYSES}

'Johan Ohlander, Samuel Fuhrimann, Martie van Tongeren, Roel Vermeulen, Anke Huss, Hans Kromhout. 'Utrecht University, Netherlands

\subsection{6/OEM-2021-EPI.48}

Introduction Assessment of occupational pesticide exposure in epidemiological studies of chronic diseases is particularly challenging. Biomonitoring of current pesticide levels might not correlate with past exposure relevant to disease etiology, and indirect methods often rely on workers' imperfect recall of exposures, or workers' job titles.

\section{Objectives}

Within the IMPRESS project (www impress-project.org) we investigated how exposure assessment method (EAM) type for assessing occupational pesticide exposure influenced risk estimates for some chronic diseases.

Methods In three meta-analyses the influence of type of EAM on the pooled risk of prostate cancer (25 articles), Non-Hodgkin's lymphoma (NHL) (29 articles), and Parkinson's disease (PD) (34 articles) was investigated using subgroup analyses by type of EAM. Data were mainly obtained from a previous systematic review conducted by the authors. Categories of EAM types used were: group-level assessments (e.g. job titles), self-reported exposures, expert-level assessments (e.g. job-exposure matrices), and biomonitoring. Further sub-group analyses were made by study design and publication year.

Results EAM types were not associated with statistically significantly different pooled risk estimates regarding any health outcome. However, for all health outcomes, case-control studies showed consistently higher risk estimates when expert-level assessments were used compared with self-reports. Overall, case-control designs showed higher risk estimates than cohort designs. Cancer studies showed higher risk estimates in later publications, whereas PD studies showed higher risk estimates in earlier publications.

Conclusion Overall, EAM type in studies of occupational pesticide exposure appears not to affect risk estimates of prostate cancer, NHL, and PD. Nevertheless, in case-control settings self-reported exposures might yield lower risk estimates than expert-based methods, possibly resulting from a higher degree of exposure misclassification due to workers imperfect recall of exposures. In systematic reviews of health effects of occupational exposure to pesticides, study design, year of publication, and exposure assessment method(s) should be taken into account. 